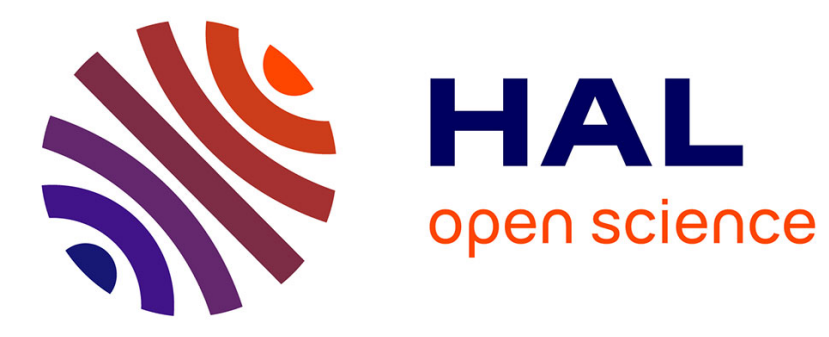

\title{
Test Statistic and Position Error Correlation in (Advanced) RAIM
}

Eugene Bang, Carl Milner, Elisa Gallon, Boris Pervan, Christophe Macabiau, Philippe Estival

\section{To cite this version:}

Eugene Bang, Carl Milner, Elisa Gallon, Boris Pervan, Christophe Macabiau, et al.. Test Statistic and Position Error Correlation in (Advanced) RAIM. ION GNSS+ 2019, 32nd International Technical Meeting of the Satellite Division of The Institute of Navigation, Sep 2019, Miami, United States. pp.534-551, 10.33012/2019.16894 . hal-02318333

\section{HAL Id: hal-02318333 \\ https://hal-enac.archives-ouvertes.fr/hal-02318333}

Submitted on 17 Aug 2020

HAL is a multi-disciplinary open access archive for the deposit and dissemination of scientific research documents, whether they are published or not. The documents may come from teaching and research institutions in France or abroad, or from public or private research centers.
L'archive ouverte pluridisciplinaire HAL, est destinée au dépôt et à la diffusion de documents scientifiques de niveau recherche, publiés ou non, émanant des établissements d'enseignement et de recherche français ou étrangers, des laboratoires publics ou privés. 


\title{
Test Statistic and Position Error Correlation in (Advanced) RAIM
}

\author{
Eugene Bang ${ }^{1}$, Carl Milner ${ }^{1}$, Elisa Gallon ${ }^{2}$, Boris Pervan ${ }^{2}$, Christophe Macabiau $^{1}$, Philippe Estival ${ }^{3}$ \\ ${ }^{1}$ Ecole Nationale de l'Aviation Civile (ENAC), France \\ ${ }^{2}$ Illinois Institute of Technology, US Illinois Institute of Technology, US \\ ${ }^{3}$ DSNA, France
}

\section{BIOGRAPHY (IES)}

Dr. Eugene Bang received his PhD degree in Aerospace Engineering from Korea Advanced Institute of Science and Technology (KAIST), Republic of Korea, in 2017. He currently works as a postdoctoral research associate at Ecole Nationale de l'Aviation Civile (ENAC) in Toulouse, France. His areas of interest include high integrity applications of GNSS and advanced receiver autonomous integrity monitoring (ARAIM). He is a recipient of the Institute of Navigation (ION) GNSS+ Student Paper Award (2016).

Dr. Carl Milner is an Assistant Professor within the Telecom Lab at the Ecole Nationale de l'Aviation Civile. He has a Masters degree in Mathematics from the University of Warwick, a PhD in Geomatics from Imperial College London and has completed the graduate trainee programme at the European Space Agency. His research interests include GNSS augmentation systems, integrity monitoring, air navigation and applied mathematics.

Dr. Christophe Macabiau graduated as an electronics engineer in 1992 from ENAC (Ecole Nationale de l'Aviation Civile) in Toulouse, France. Since 1994, he has been working on the application of satellite navigation techniques to civil aviation. He received his $\mathrm{Ph} . \mathrm{D}$ in 1997 and has been in charge of the signal processing lab of ENAC since 2000, where he also started dealing with navigation techniques for terrestrial navigation. He is currently the head of the TELECOM team of ENAC, that includes research groups on signal processing and navigation, electromagnetics, and data communication networks.

Philippe ESTIVAL is a GNSS expert at the French civil aviation service provider (DGAC/DSNA/DTI) currently involved in EGNOS mission requirements and standardization activities on future multi-constellation GNSS receivers Eurocae WG62 and within EU/US cooperation agreement Working Group C. He has been in charge of CCF (EGNOS Central Control Facility) operational support and evolutions for PACF department until 2009 and then for ESSP SAS System Operation Unit up to 2015. He graduated in 2005 as an electronics engineer from the Ecole Nationale de l'Aviation Civile (ENAC) in Toulouse, France

Elisa Gallon received her Bachelor's Degree in Mathematics from Université Blaise Pascal, France in 2014 and her Master of Science in Global Navigation Satellite Systems from ENAC and ISAE-Supaéro in 2016. From 2016 to 2017, she worked at the European Space Agency (ESA) on Orbit Determination and Time Synchronization of Galileo satellites. She is currently a Ph.D. Candidate at the Navigation Laboratory in the Department of Mechanical and Aerospace Engineering at Illinois Institute of Technology (IIT) in Chicago.

Dr. Boris Pervan is a Professor of Mechanical and Aerospace Engineering at IIT, where he conducts research on advanced navigation systems. Prior to joining the faculty at IIT, he was a spacecraft mission analyst at Hughes Aircraft Company (now Boeing) and a postdoctoral research associate at Stanford University. Prof. Pervan received his B.S. from the University of Notre Dame, M.S. from the California Institute of Technology, and Ph.D. from Stanford University. He is an Associate Fellow of the AIAA, a Fellow of the Institute of Navigation (ION), and Editor-in-Chief of the ION journal NAVIGATION. He was the recipient of the IIT Sigma Xi Excellence in University Research Award (2011, 2002), Ralph Barnett Mechanical and Aerospace Dept. Outstanding Teaching Award (2009, 2002), Mechanical and Aerospace Dept. Excellence in Research Award (2007), University Excellence in Teaching Award (2005), IEEE Aerospace and Electronic Systems Society M. Barry Carlton Award (1999), RTCA William E. Jackson Award (1996), Guggenheim Fellowship (Caltech 1987), and Albert J. Zahm Prize in Aeronautics (Notre Dame 1986). 


\begin{abstract}
Previous investigation on the measurement temporal correlation effect on Probability of Hazardously Misleading Information (PHMI) [1] has proposed how to map the operational integrity risk to that for a single sample. However, a well-known assumption that the stochastic components of position error and the test statistic are independent was taken to evaluate the actual risk over a given time interval. This paper examines the level of cross-correlation present between the estimated states and the fault detection statistics for (Advanced) Receiver Autonomous Integrity Monitoring (RAIM) and determines its impact on the PHMI to generalize the proposed approach. In this study, we take several experimental and simulation methods. Dual-frequency GPS and Galileo measurements were collected and processed to measure the correlation between the position error and monitor test statistic. We also evaluate actual integrity risk when they are correlated through using simple satellite geometries and error models as well as those for H-ARAIM applications. It was found that the cross-correlation between the position and test domains is present when the measurement error inflation model, e.g., commonly used Gaussian overbounding method, is applied to the weighted least-squares estimation. Also, the numerical results of the integrity risk evaluation showed that the assumption taken in the previous assessment is valid with respect to correlation resulting from sigma inflation even though the additional risk of up to $60 \%$ is observed due to the correlation effect.
\end{abstract}

\title{
INTRODUCTION
}

Receiver Autonomous Integrity Monitoring (RAIM) was developed in the 1980s as a means to support horizontal guidance for enroute operations. RAIM is based on two primary functions; fault detection and integrity assessment using protection levels. A third function, fault exclusion, may also be used to improve continuity performance [2]. RAIM fault detection methods were developed primarily using a snapshot approach, employing measurements from the current epoch alone. The most commonly presented techniques are least-squares residuals based [3], parity vector based [4] and solution separation based [5]. In terms of fault detection, there is some equivalence between methods as shown in [6]. They may be nominally, if not exhaustively, classified by those which employ a single chi-squared test for all fault modes and those which employ individual Gaussian tests for each fault mode [7]. The (weighted) norm of the residuals vector may be used in the former manner or alternatively the normalized residuals vector elements in the latter [6], [8]. Similarly the norm of the parity vector equates to the residuals norm but also the parity vector may be projected on to the characteristic satellite fault lines by taking the inner product and forming scalar Gaussian statistics [6]. Finally, the solution separation approach forms Gaussian statistics which were used to test single satellite faults [5] and relate to the parity projections [7].

It should be noted that the validity of Gaussian and Chi-Squared here is subject to the underlying measurement error model and will be discussed in greater detail below. In addition to the hypothesis tests for fault detection (and exclusion) RAIM computes a protection level to be compared to the alert limit in order to determine if the integrity requirements are met. Two methods are commonly used, a slope based approach which maps a minimum detectable bias from the test statistic domain to the position domain before applying an additional safety buffer [9], and the solution separation protection level employing the triangle inequality [10].

Recently, the Advanced Receiver Autonomous Integrity Monitoring (ARAIM) concept has been proposed as a means to guarantee Required Navigation Performance (RNP) with 0.1NM bounds and then, to support Localizer Performance with Vertical guidance (LPV) down to 200ft with global coverage. ARAIM employs a Multiple Hypothesis Solution Separation (MHSS) approach [11] [12] [13]. Recent work on ARAIM has investigated the means to map integrity risk over an operation (exposure time) to the integrity risk for a sample at algorithmic level [1]. This work employed the result given in [14], that the stochastic components of the estimated state vector and the test metrics are independent. However, it is noted that this only remains valid when the assumed Gaussian measurement model exactly characterizes the true errors. In reality a Gaussian overbound is used which as well as employing the simple form of the Gaussian, also inflates the standard deviation to ensure a margin of conservatism. This inflation effectively means that the weighted least squares solution is sub-optimal and leading to a correlation.

Note that the solution separation protection level does not require independence of the test and state vector since the integrity bounding is based on the properties of the single subset position error not on the pair of processes [15]. However, slope based RAIM and the assessment performed in [1] to support ARAIM developments with regards to temporal correlation are reliant on this relationship. Therefore, this paper addresses the level of correlation between the estimated states and the test statistic that may occur due to error model inflation. Four different techniques are used to make this assessment as given in the paper outline below. 
The paper is organized as follows. Firstly, the relevant (A)RAIM fundamentals are presented. The paper then treats some canonical examples to confirm the presence of position error to test matric correlation resulting from model inflation and get a handle on the magnitude of its impact. Secondly, real data is analyzed to measure the correlation as a result of the real error model inflation using the IIT ARAIM prototype. Finally, real geometries are employed with simulated measurement errors employing inflated models in order to compare to the real data.

\section{AUTONOMOUS INTEGRITY}

\section{Linear Model}

The paper assumes the following linearized measurement model after resolution of the iterative weighted least squares estimation (WLSE):

$$
\mathbf{z}=\mathbf{H x}+\boldsymbol{\varepsilon}
$$

where

$\mathbf{Z}$

is the observed $n \times 1$ linearized measurement vector obtained from ionosphere-free carrier smoothed code and subtracted the predicted measurement based on the final estimated solution

$\boldsymbol{H} \quad$ is the known $n \times m$ observation matrix representing the satellite-user geometry. It is formed from the Jacobian of the non-linear model [3] and is purely a function of the satellite azimuths and elevations

$x$

is the unknown $m \times 1$ state vector comprising of east-north-up position states in the local frame and constellationreceiver clock offset states

$\boldsymbol{\varepsilon} \quad$ is the observed $n \times 1$ linearized measurement error vector

The error vector is assumed under Weighted RAIM and ARAIM to be composed of a fault vector component, a nominal bias with maximal magnitude and a stochastic (noise) component.

$$
\varepsilon=\mu+b_{\text {nom }}+v
$$

where:

$\begin{array}{ll}\boldsymbol{\mu} & \text { is the } n \times 1 \text { linearized measurement fault vector } \\ \boldsymbol{b}_{\text {nom }} & \text { is the measurement error nominal bias vector } \\ \boldsymbol{v} & \text { is the zero-mean measurement error noise vector }\end{array}$

Both $\boldsymbol{\mu}$ and $\boldsymbol{b}_{\text {nom }}$ are assumed to be deterministic quantities whilst the noise components are assumed to be conservatively bounded by a zero-mean Gaussian.

where

$$
\boldsymbol{v} \sim N\left(\mathbf{0}, \boldsymbol{\Sigma}_{\varepsilon}\right)
$$

$$
\boldsymbol{\Sigma}_{\varepsilon}=\left(\begin{array}{ccc}
\sigma_{1}^{2} & \cdots & 0 \\
\vdots & \ddots & \vdots \\
0 & \cdots & \sigma_{n}^{2}
\end{array}\right)
$$

The variances are constructed for the ARAIM as:

$$
\sigma_{i}^{2}=\sigma_{U R A, i}^{2}+\sigma_{\text {tropo }, i}^{2}+\sigma_{\text {user }, i}^{2}
$$

Or for the more realistic accuracy and continuity purposes: 


$$
\sigma_{i}^{2}=\sigma_{U R E, i}^{2}+\sigma_{\text {tropo }, i}^{2}+\sigma_{u s e r, i}^{2}
$$

where typically [16]:

$$
\frac{\sigma_{U R E, i}}{\sigma_{U R A, i}} \approx \frac{2}{3}
$$

For the traditional single-frequency based RAIM, an ionospheric overbounding term, $\sigma_{\text {iono }}$, should be considered in addition to error variances in Equation (5) to construct the measurement error model.

$$
\sigma_{i}^{2}=\sigma_{U R A, i}^{2}+\sigma_{\text {iono }, i}^{2}+\sigma_{\text {tropo }, i}^{2}+\sigma_{\text {user }, i}^{2}
$$

Current assumptions regarding the magnitude of the nominal bias under ARAIM are that it is bounded by $75 \mathrm{~cm}$ while it is set to zero under the RAIM. Employing WLSE, the estimated states are obtained as follows:

$$
\boldsymbol{x}_{\mathbf{0}}=\left(\boldsymbol{H}^{T} \boldsymbol{W H}\right)^{-1} \boldsymbol{H}^{T} \boldsymbol{W} \mathbf{z}=\boldsymbol{S}_{0} \boldsymbol{z}
$$

where

$$
\mathbf{W}=\Sigma_{\varepsilon}^{-1}
$$

\section{Fault Detection Tests}

In this paper, the solution separation and parity tests will be used. The solution separation tests are defined as:

$$
q_{c i}=\frac{\boldsymbol{\alpha}^{T} \boldsymbol{\Delta} \boldsymbol{x}_{i}}{\sigma_{s s, c i}}=\frac{\boldsymbol{\alpha}^{T}\left(\boldsymbol{S}_{i}-\boldsymbol{S}_{0}\right) \mathbf{z}}{\sigma_{s s, c i}}
$$

where

$\boldsymbol{S}_{i}=\left(\boldsymbol{H}^{T} \boldsymbol{W}_{\boldsymbol{i}} \boldsymbol{H}\right)^{-1} \boldsymbol{H}^{T} \boldsymbol{W}_{\boldsymbol{i}}$

$\boldsymbol{\alpha}^{T} \quad$ is the coordinate selection vector i.e. [1 ... 0 ] would select the first state vector element

$c \quad$ is the index for the coordinate such as East, North and Up direction

$\sigma_{s s, c i} \quad$ is the standard deviation of the numerator, the un-normalized test

A threshold for detection is determined using the following relation.

$$
k=Q^{-1}\left(P_{f a, c i}\right)
$$

where $Q$ is the right-tail probability of a zero mean unit normal distribution, defined as

$$
Q(k)=\frac{1}{\sqrt{2 \pi}} \int_{k}^{\infty} e^{-\frac{z^{2}}{2}} d z
$$

The parity vector may be used as an equivalent test.

$$
\boldsymbol{p}=\boldsymbol{P z}
$$

where 


$$
\boldsymbol{P}=\boldsymbol{U}_{2}^{T}, \boldsymbol{H}=\boldsymbol{U} \boldsymbol{D} \boldsymbol{V}^{T}=\left[\begin{array}{ll}
\boldsymbol{U}_{1} & \boldsymbol{U}_{2}
\end{array}\right]\left[\begin{array}{c}
\boldsymbol{D}_{1} \\
\mathbf{0}
\end{array}\right] \boldsymbol{V}^{T}=\boldsymbol{U}_{1} \boldsymbol{D}_{1} \boldsymbol{V}^{T}
$$

The method of Single Value Decomposition provides an upper triangular matrix $\boldsymbol{D}_{\mathbf{1}} \boldsymbol{V}^{T}$ and an orthogonal matrix $\boldsymbol{U}$.

$$
q=\frac{\boldsymbol{p}^{T} \boldsymbol{P}_{i}}{\left\|\boldsymbol{P}_{i}\right\|}
$$

where:

$\boldsymbol{P}_{i} \quad$ is column relating to a single satellite

\section{Independence Under Optimal Model}

In reference [14], a normalized linear model is employed, which will be the case when addressing the canonical examples in the later section. In this section, the model is assumed to have been pre-normalized such that

$$
\mathbf{z}^{*}=\boldsymbol{H}^{*} \boldsymbol{x}+\boldsymbol{\varepsilon}^{*}
$$

where

$z^{*}=w^{\mathbf{1} / \mathbf{2}} Z$ is the normalized measurement vector

$\boldsymbol{H}^{*}=\boldsymbol{w}^{\mathbf{1 / 2}} \boldsymbol{H}$ is the normalized observation matrix

$\boldsymbol{\varepsilon}^{*}=\boldsymbol{w}^{\mathbf{1 / 2} \boldsymbol{\varepsilon}}$ is the normalized measurement error vector

$\boldsymbol{w}^{\mathbf{1} / 2}=\left[\begin{array}{lll}1 / \sigma_{1} & \cdots & 1 / \sigma_{n}\end{array}\right]$

With this transformation in place the error covariance is the identity.

$$
E\left[\varepsilon^{*} \varepsilon^{* T}\right]=I_{n}
$$

The test is then naturally defined to be formed on the basis of a linear combination of the measurements (i.e. residuals vector for the LSR method).

$$
q=Q z
$$

Since $\boldsymbol{Q z}$ is providing a direct observation of the fault vector $\boldsymbol{f}$ it is argued that:

$$
Q H=0
$$

which is equivalent to $\boldsymbol{Q}$ mapping to the null space of $\boldsymbol{H}^{T}$. The correlation between test vector (i.e. parity vector) and the estimated state vector is given as:

$$
E\left[Q z e^{T}\right]=E\left[Q \varepsilon \varepsilon^{T} s\right]=Q s=Q H P \alpha=0
$$

where the property of Equation (20) has been used. However, this is only true if the weights used to populate $\boldsymbol{w}^{\mathbf{1} / \mathbf{2}}$ truly reflect the standard deviations of the errors $\boldsymbol{\varepsilon}$. If the errors are non-Gaussian or have truly different standard deviations the above relation is not sure to be zero and as is seen below will be non-zero if the scaling of assumed values to reality is not identical for all measurements.

\section{Correlation and its Impact}

Ultimately, the ratio of the inflated but uncorrelated risk, and the uninflated yet correlated risk, is the key metric of the impact of inflation on correlation. 


$$
r(k, l)=\frac{P\left(e>l \& q<k \mid \boldsymbol{\Sigma}_{\varepsilon}{ }^{\prime}, \boldsymbol{H}, \boldsymbol{W}=\boldsymbol{\Sigma}_{\varepsilon}{ }^{-1}\right)}{P\left(e>l \& q<k \mid \boldsymbol{\Sigma}_{\varepsilon}, \boldsymbol{H}, \boldsymbol{W}=\boldsymbol{\Sigma}_{\varepsilon}{ }^{-1}\right)}
$$

Here $e$ denotes the position error and $k$ and $l$ respectively indicate detection threshold and the protection level or alert limit. Note that in both the numerator and denominator the same geometry matrix and assumed weighting is employed but the first conditional parameter differs, relating to the assumed error performance. Elements of $\boldsymbol{\Sigma}_{\boldsymbol{\varepsilon}}{ }^{\prime}$ will be no larger than elements of $\boldsymbol{\Sigma}_{\varepsilon}$ and as a result of inflation may be smaller. This expression $r(k, l)$ is a function of the threshold $(k)$ and the protection bound or alert limit $(l)$. With Equation (22) in place, the following sections will present methods to determine the level of correlation and the impact on the risk ratio.

\section{CANONICAL EXAMPLES}

In the following examples the linear models are assumed to have been pre-normalized and as such the inflated covariance matrices are given by the identity matrix in each case. Uneven variances are treated in the real data and simulated data techniques which follows.

\section{Example 1}

In this first example, the simplest possible observation matrix is used:

$$
\boldsymbol{H}=\left[\begin{array}{l}
1 \\
1
\end{array}\right]
$$

With assumed measurement covariance matrix:

$$
\boldsymbol{\Sigma}_{\varepsilon}=\left(\begin{array}{ll}
1 & 0 \\
0 & 1
\end{array}\right)
$$

The pseudoinverse is then:

$$
\boldsymbol{S}=\left[\begin{array}{ll}
1 / 2 & 1 / 2
\end{array}\right]
$$

The parity domain is 1D such that only a single test may be defined using the parity matrix (obtained through Single Value Decomposition) as [7]:

$$
\boldsymbol{P}=\left[\begin{array}{ll}
-1 / \sqrt{2} & 1 / \sqrt{2}
\end{array}\right]
$$

Note that $\boldsymbol{P H}=\mathbf{0}$ as expected from [14]. The state estimation error and the test are then obtained as:

$$
\begin{gathered}
e=\boldsymbol{S} \boldsymbol{\varepsilon}=\left[\begin{array}{ll}
1 / 2 & 1 / 2
\end{array}\right]\left[\begin{array}{l}
\varepsilon_{1} \\
\varepsilon_{2}
\end{array}\right]=\frac{\varepsilon_{1}+\varepsilon_{2}}{2} \\
q=\boldsymbol{P} \boldsymbol{\varepsilon}=\left[\begin{array}{ll}
-1 / \sqrt{2} & 1 / \sqrt{2}
\end{array}\right]\left[\begin{array}{l}
\varepsilon_{1} \\
\varepsilon_{2}
\end{array}\right]=\frac{\varepsilon_{2}-\varepsilon_{1}}{\sqrt{2}}
\end{gathered}
$$

We consider the impact of a ranging fault bias which introduces a bias $\mu_{q}$ on $q$ and $\mu_{e}$ on $e$ and define two normalized variables:

$$
z_{e}=\frac{e-\mu_{e}}{\sigma_{e}}
$$




$$
z_{q}=\frac{q-\mu_{q}}{\sigma_{q}}
$$

The standard deviations are obtained as follows:

$$
\begin{gathered}
\sigma_{e}=\sqrt{\left(\boldsymbol{H}^{\boldsymbol{T}} \boldsymbol{H}\right)^{-1}}=\sqrt{\boldsymbol{S \boldsymbol { S } ^ { T }}}=\frac{1}{\sqrt{2}} \\
\sigma_{q}=\sqrt{\boldsymbol{P} \boldsymbol{P}^{T}}=1
\end{gathered}
$$

We allow $e, q, \mu_{e}, \mu_{q}, \sigma_{e}$ and $\sigma_{q}$ to take any values such that $z_{e}$ and $z_{q}$ lie between -6 and 6 . Since it is assumed that $e$ and $q$ are Gaussian variables, the alternative occurs with a negligible probability $\left(<10^{-9}\right)$. The risk ratio given in Equation $(22)$ may then be restated as:

$$
r(k, l)=\frac{P\left(z_{q}^{\prime}>k_{q} \& z_{e}^{\prime}>k_{e} \mid \boldsymbol{\Sigma}_{\varepsilon}{ }^{\prime}, \boldsymbol{H}, \boldsymbol{W}=\boldsymbol{\Sigma}_{\varepsilon}{ }^{-1}\right)}{P\left(z_{q}>k_{q} \& z_{e}>k_{e} \mid \boldsymbol{\Sigma}_{\varepsilon}, \boldsymbol{H}, \boldsymbol{W}=\boldsymbol{\Sigma}_{\varepsilon}{ }^{-1}\right)}
$$

For two thresholds $k_{e}$ and $k_{q}$ between -6 and 6 , the following quantities are computed. Note that the without loss of generality, the reverse inequality condition is used for $q$. Results relating to the test being below the threshold are obtained by simply reflecting the $\mathrm{x}$-axis of Figure 1 below.

Firstly, the inflated but uncorrelated probability.

$$
P\left(z_{q}>k_{q} \& z_{e}>k_{e} \mid \boldsymbol{\Sigma}_{\varepsilon}\right)=Q\left(k_{q}\right) Q\left(k_{e}\right)
$$

Secondly, the uninflated yet correlated risk.

$$
P\left(z_{q}{ }^{\prime}>k_{q} \& z_{e}{ }^{\prime}>k_{e} \mid \Sigma_{\varepsilon}{ }^{\prime}\right)=Q_{b i}\left(k_{q}, k_{e} ; \rho\right)
$$

is the bivariate normalized Gaussian distribution with correlation $\rho$. Note that here, the Gaussian variables $z_{q}{ }^{\prime}$ and $z_{e}{ }^{\prime}$ are normalized using the standard deviations $\sigma_{q}{ }^{\prime}$ and $\sigma_{e}{ }^{\prime}$ obtained from the uninflated measurement model with covariance $\boldsymbol{\Sigma}_{\varepsilon}{ }^{\prime}$. This covariance matrix is obtained by inverting the error model inflation process. With inflation factors $\boldsymbol{\alpha}=\left[\begin{array}{ll}\alpha_{1} & \alpha_{2}\end{array}\right]$,

$$
\Sigma_{\varepsilon}{ }^{\prime}=\Sigma_{\varepsilon} \times \operatorname{diag}(\alpha)^{-1}
$$

The correlation factor, as well as the covariances of estimated states and parity matrix are then given as:

$$
\begin{aligned}
\boldsymbol{\Sigma}_{e}{ }^{\prime} & =\boldsymbol{S} \boldsymbol{\Sigma}_{\varepsilon}{ }^{\prime} \boldsymbol{S}^{T} \\
\boldsymbol{\Sigma}_{q}{ }^{\prime} & =\boldsymbol{P} \boldsymbol{\Sigma}_{\varepsilon}{ }^{\prime} \boldsymbol{P}^{T} \\
\boldsymbol{\Sigma}_{\boldsymbol{q}}{ }^{\prime} & =\boldsymbol{P} \boldsymbol{\Sigma}_{\varepsilon}{ }^{\prime} \boldsymbol{S}^{T} \\
\sigma_{e}^{\prime} & =\sqrt{\boldsymbol{\Sigma}_{e}^{\prime}} \\
\sigma_{q}^{\prime} & =\sqrt{\boldsymbol{\Sigma}_{q}^{\prime}} \\
\rho & =\boldsymbol{\Sigma}_{q e}^{\prime}
\end{aligned}
$$

Only the relative inflation is relevant, since equal inflation factors simply factor out of the WLSE expressions. In this example $\alpha_{1}=$ 1 without loss of generality. With $\alpha_{2}=1.5$ which relates roughly to the GNSS case of the URA to URE (or SISA to SISE) ratio, the 
correlation $\rho$ is approximately -0.2. In Figure 1, the computed probabilities in logarithm base ten scale using Equation (34) are shown over the 2D range from -6 to 6 .

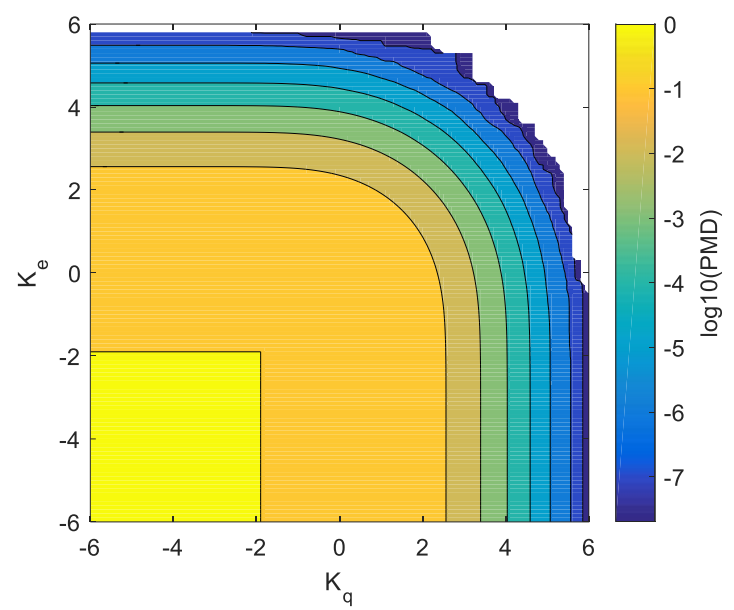

Figure 1. $\log _{10}(\mathrm{P})$ over $\left(k_{q}, k_{e}\right) \in[-6,6]^{2}$

In Figure 2 below the ratio in probabilities is given between expressions (33) and (37). Note that in Figure 2 the ratio does not exceed unity, and as such the use of inflated sigmas does lead to an underestimated probability of crossing both thresholds when failing to account for the correlation.

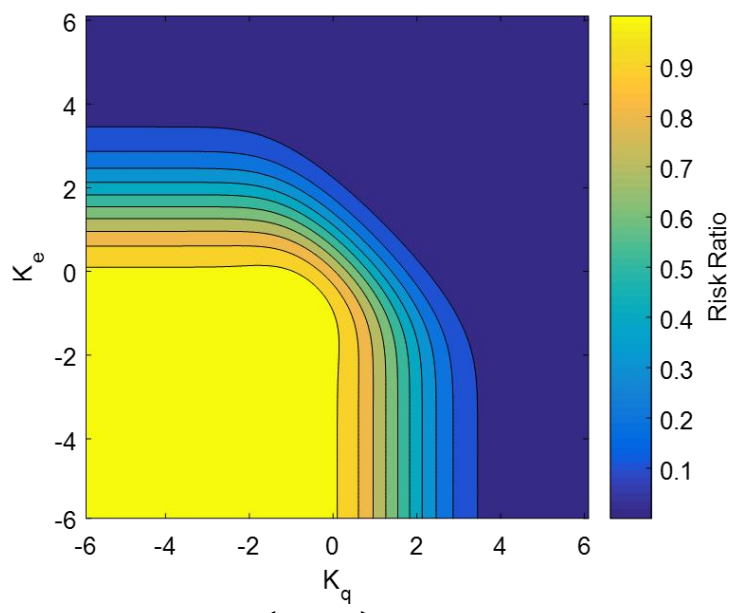

Figure 2. Risk ratio over $\left(k_{q}, k_{e}\right) \in[-6,6]^{2}$ for $\sigma_{2}$ inflation of 1.5

However, if $\alpha_{2}=4$ which represents an extreme case of inflation, where $\rho=-0.33$, Figure 3 now shows the resulting ratios which exceed unity. In this example, $\alpha_{2}$ of 4 is determined based on the previous observations from [17] where the maximum inflation between the minimum overbounding URA and the broadcast URA for the GPS satellite is up to around 4. It is important to note, however, that this high ratio is for situations where the probability of occurrence is high (greater than $10^{-2}$ ). Since the validity of RAIM and ARAIM protection levels is assessed with the dominant fault modes at probabilities in the range from $10^{-3}$ down to $10^{-8}$ it might be argued that this result is of no relevance and the inflation does not introduce additional risk when assuming independence between the two variables $q$ and $e$. 


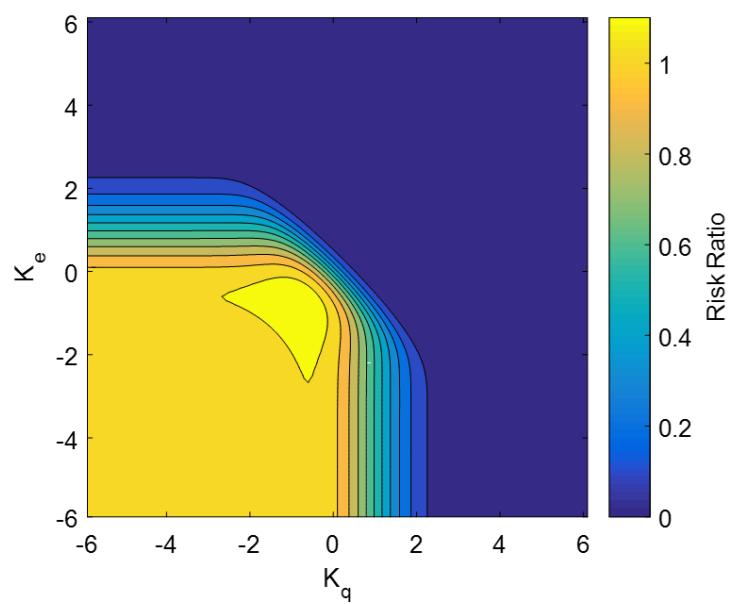

Figure 3. Risk ratio over $\left(k_{q}, k_{e}\right) \in[-6,6]^{2}$ for $\sigma_{2}$ inflation of 4.0

Next, to assess the worst case a method based on convex optimization is used to conclude on a maximum inflation factor of the integrity risk as a result of the correlation. Since the uninflated covariance $\left(\boldsymbol{\Sigma}_{\varepsilon}^{\prime}\right)$ is a function of the inflation vector, $\boldsymbol{\alpha}$, the worst-case inflation can be identified such that the risk ratio is maximized at the corresponding inflation. Thus, we can cast the simple example up to an extreme-inflation factor of 4 , to the linear inequality constrained optimization problem as follows.

$$
\begin{aligned}
r(k, l ; \alpha) & =\max _{\boldsymbol{\alpha}} \frac{P\left(z_{q}^{\prime}>k_{q} \& z_{e}^{\prime}>k_{e} \mid \boldsymbol{\Sigma}_{\varepsilon}^{\prime}(\boldsymbol{\alpha}), \boldsymbol{H}, \boldsymbol{W}=\boldsymbol{\Sigma}_{\varepsilon}{ }^{-1}\right)}{P\left(z_{q}>k_{q} \& z_{e}>k_{e} \mid \boldsymbol{\Sigma}_{\boldsymbol{\varepsilon}}, \boldsymbol{H}, \boldsymbol{W}=\boldsymbol{\Sigma}_{\boldsymbol{\varepsilon}}^{-1}\right)} \\
& \text { subject to } \boldsymbol{\alpha}=\left[1, \alpha_{2}\right] \text { where } 1<\alpha_{2} \leq 4
\end{aligned}
$$

In this case, the same upper bound for $\alpha_{2}$ in the constraint on the inflation factor is determined for the comparison with the last example.
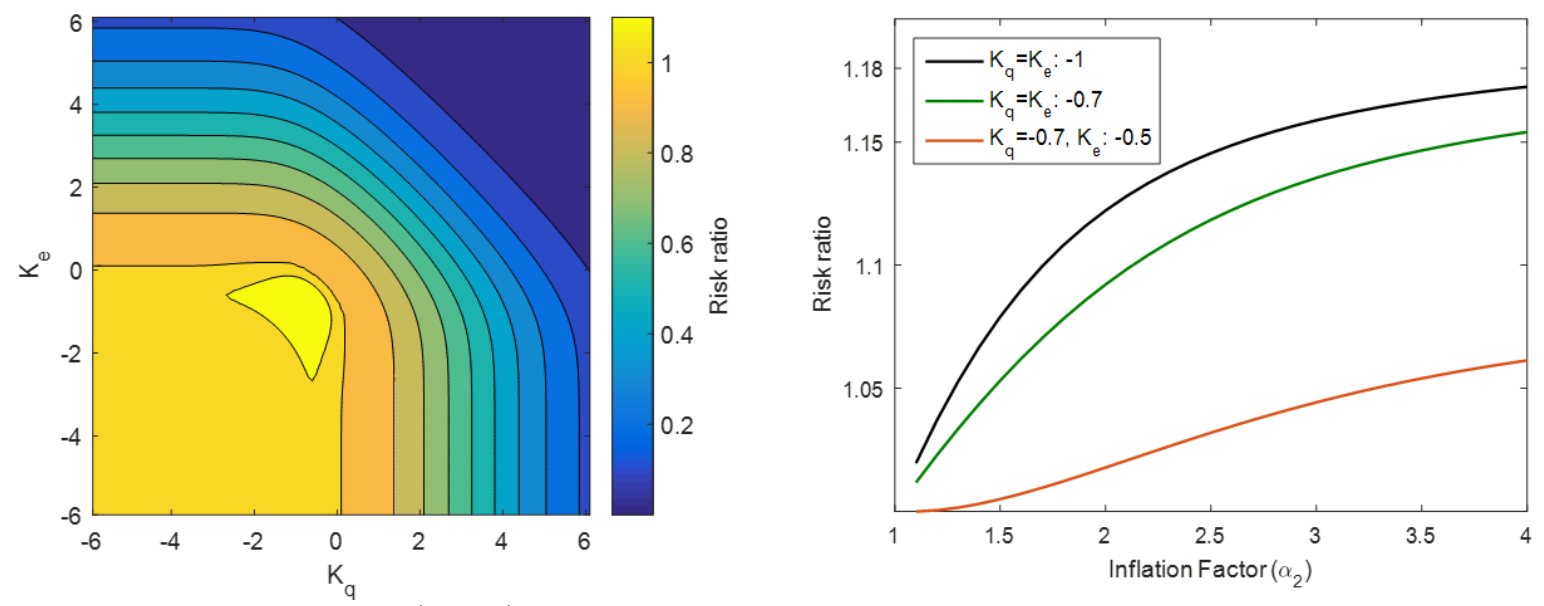

Figure 4. Maximum risk ratio over $\left(k_{q}, k_{e}\right) \in[-6,6]^{2}$ for the worst case $\sigma_{2}$ inflation (left) and example ratio curve over the inflation factor from 1.1 to 4 . 
The left in Figure 4 represents the maximum risk ratio values recomputed when the worst inflation is applied to the risk ratio calculation for different combinations of the thresholds, $k_{q}$ and $k_{e}$. The right figure shows the risk ratio over the inflation factor $\alpha_{2}$ from 1.1 to 4 for three different combinations of the thresholds corresponding to the yellow area in the risk ratio space. From the right figure, the risk ratio defined as the objective function in the problem is shown to be concave graphically. Also, it is well-known that the feasible region derived by the linear inequality constraint is convex. Thus, the global maximizer $\alpha_{2}$ and the corresponding maximum should exist for the given constraint.

As shown in the right figure, the inflation factor of up to 4 is determined for the three most critical cases where the risk ratio is higher than one, which is in line with observations from [17] for GPS satellites. On the other hand, we observed that ratio curves for other green and blue regions reach their peak at different inflations that are below 4, resulting in higher risk ratio values than the result in Figure 3. Thus, the left figure shows that the risk ratio increases in most regions when the worst inflation method is applied. However, as described in the previous example, the risk increase due to the error inflation might not affect (A)RAIM applications dramatically, even with the worst error inflation. Although a straightforward example was addressed in this paper, continuing research work will examine more general cases to determine the most significant increase of risk due to the correlation effect along with the theoretical proof of the convexity (or concavity) of the risk ratio over all possible variations of the inflation factor.

\section{Example 2}

In the second example, the number of measurements is increased to three.

$$
\boldsymbol{H}=\left[\begin{array}{l}
1 \\
1 \\
1
\end{array}\right]
$$

With assumed measurement covariance matrix:

$$
\boldsymbol{\Sigma}_{\varepsilon}=\left(\begin{array}{lll}
1 & 0 & 0 \\
0 & 1 & 0 \\
0 & 0 & 1
\end{array}\right)
$$

The pseudoinverse is then:

$$
\boldsymbol{S}=\left[\begin{array}{lll}
1 / 3 & 1 / 3 & 1 / 3
\end{array}\right]
$$

The parity domain is now $2 \mathrm{D}$. The parity matrix is [7]:

$$
\boldsymbol{P}=\sqrt{\frac{2}{3}}\left[\begin{array}{ccc}
1 & -\cos \left(\frac{\pi}{3}\right) & -\cos \left(\frac{\pi}{3}\right) \\
0 & \sin \left(\frac{\pi}{3}\right) & -\sin \left(\frac{\pi}{3}\right)
\end{array}\right]
$$

Three correlated tests may be defined either using the solution separation derivation or projection onto the columns of $\boldsymbol{P}$.

$$
q_{i}=\frac{\boldsymbol{P}_{i}{ }^{T} \boldsymbol{P} \mathbf{z}}{\left\|\boldsymbol{P}_{i}\right\|}
$$

where $\boldsymbol{P}_{i}$ is the $i^{\text {th }}$ column of $\boldsymbol{P}$. The presence of inflation factors introduces correlation between the position error and test but also within the parity domain itself. Figure 5 shows this effect with $\boldsymbol{\alpha}=\left[\begin{array}{lll}1 & 1.5 & 1\end{array}\right]$. The parity vector covariance is scaled in the direction of the un-inflated satellite 2 leading to:

$$
\boldsymbol{\Sigma}_{q}{ }^{\prime}=\left[\begin{array}{ll}
0.9074 & 0.1604 \\
0.1604 & 0.7222
\end{array}\right]
$$




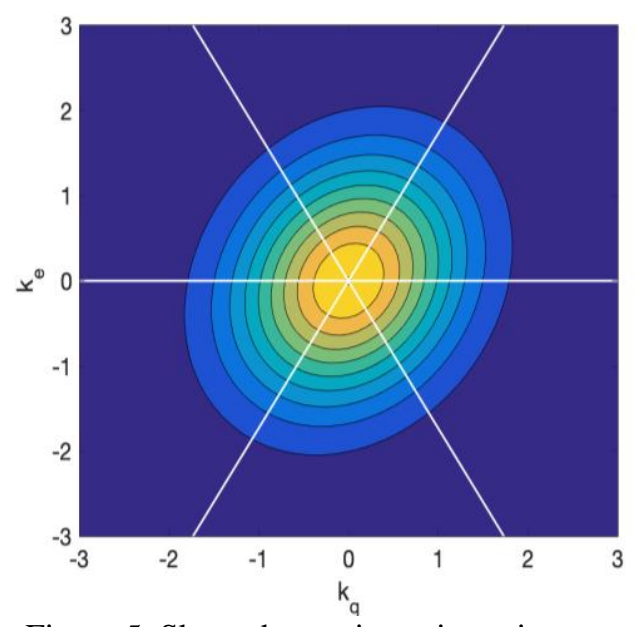

Figure 5. Skewed covariance in parity space

Figure 6 and Figure 7 show the risk ratio of Equation (33) over the normalized position error and test metric space for the case of $q_{2}$ (note the measurement with inflated variance) whilst Figure 8 and 9 shows the ratio for the two remaining metrics $q_{1}$ and $q_{3}$.
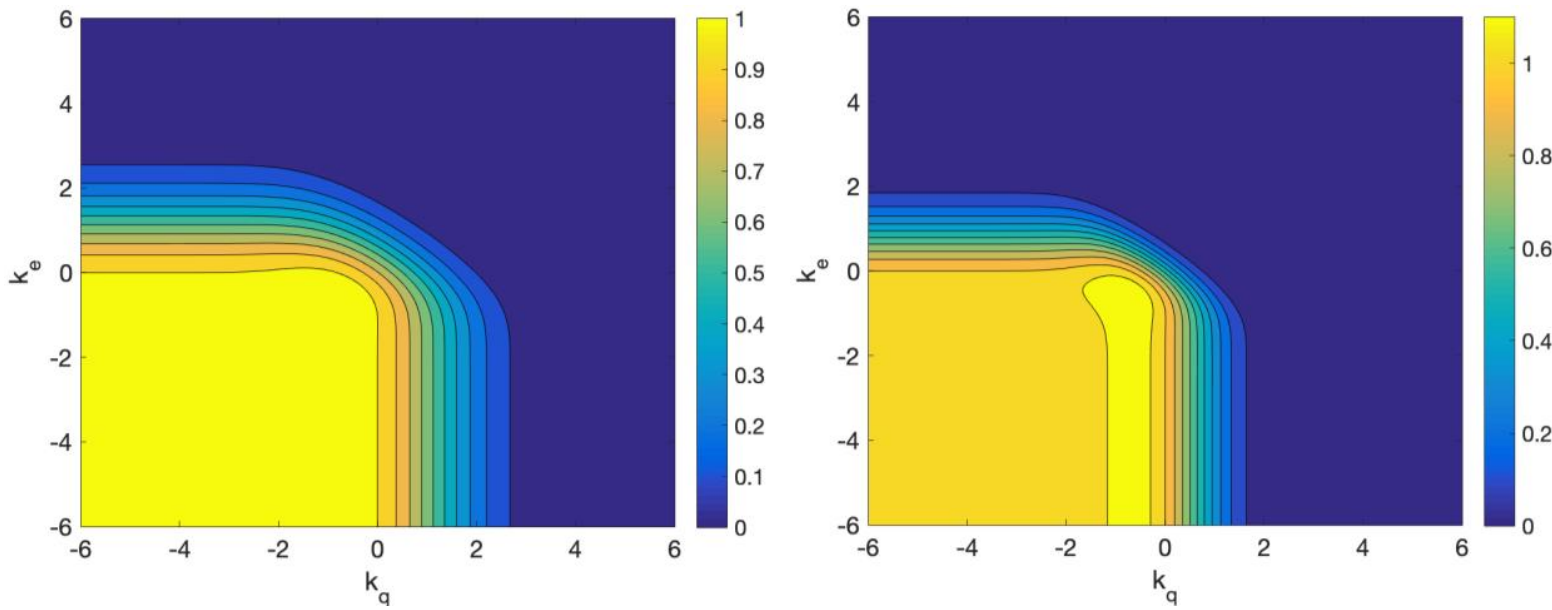

Figure 6. Risk ratio for $q_{2}$ for $\sigma_{2}$ inflation of 1.5 (left) and 3.0 (right)
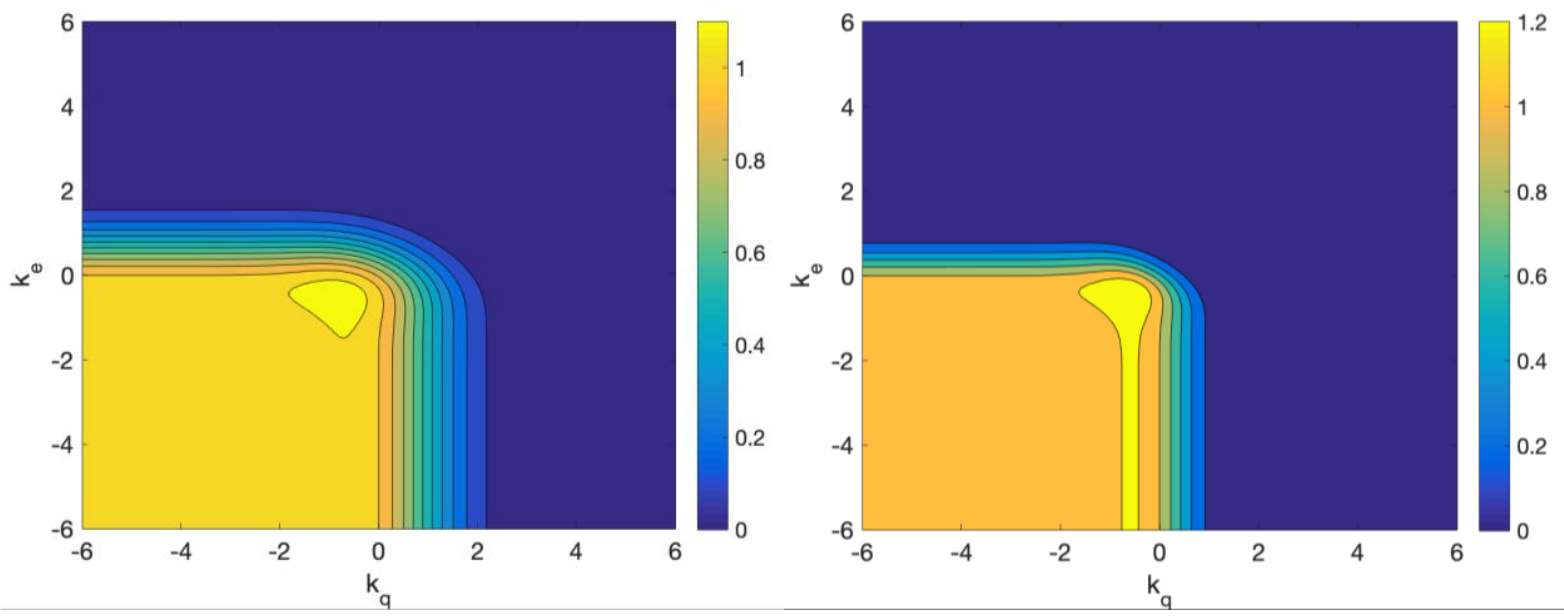

Figure 7. Ratio for $q_{2}$ and $q_{3}$ for $\sigma_{2}$ and $\sigma_{3}$ inflation of 1.5 (left) and 3.0 (right) 

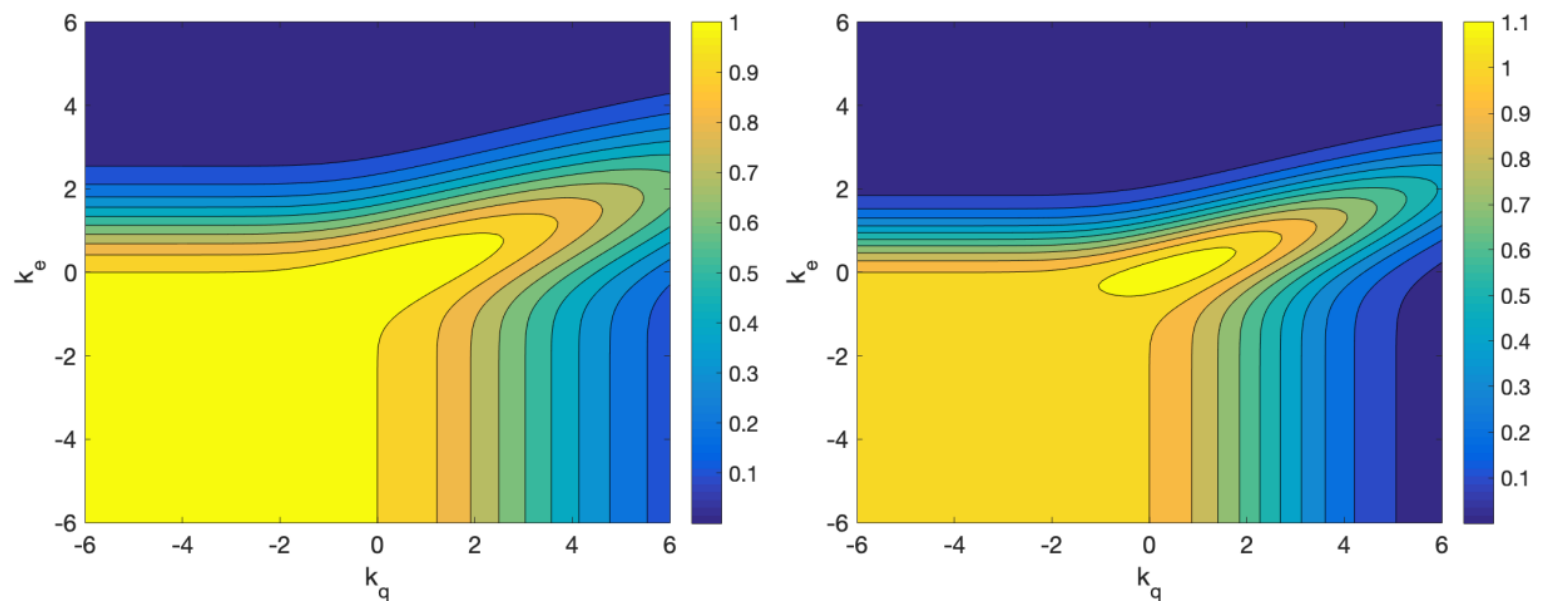

Figure 8. Risk ratio for $q_{1}$ and $q_{3}$ for $\sigma_{2}$ inflation of 1.5 (left) and 3.0 (right)
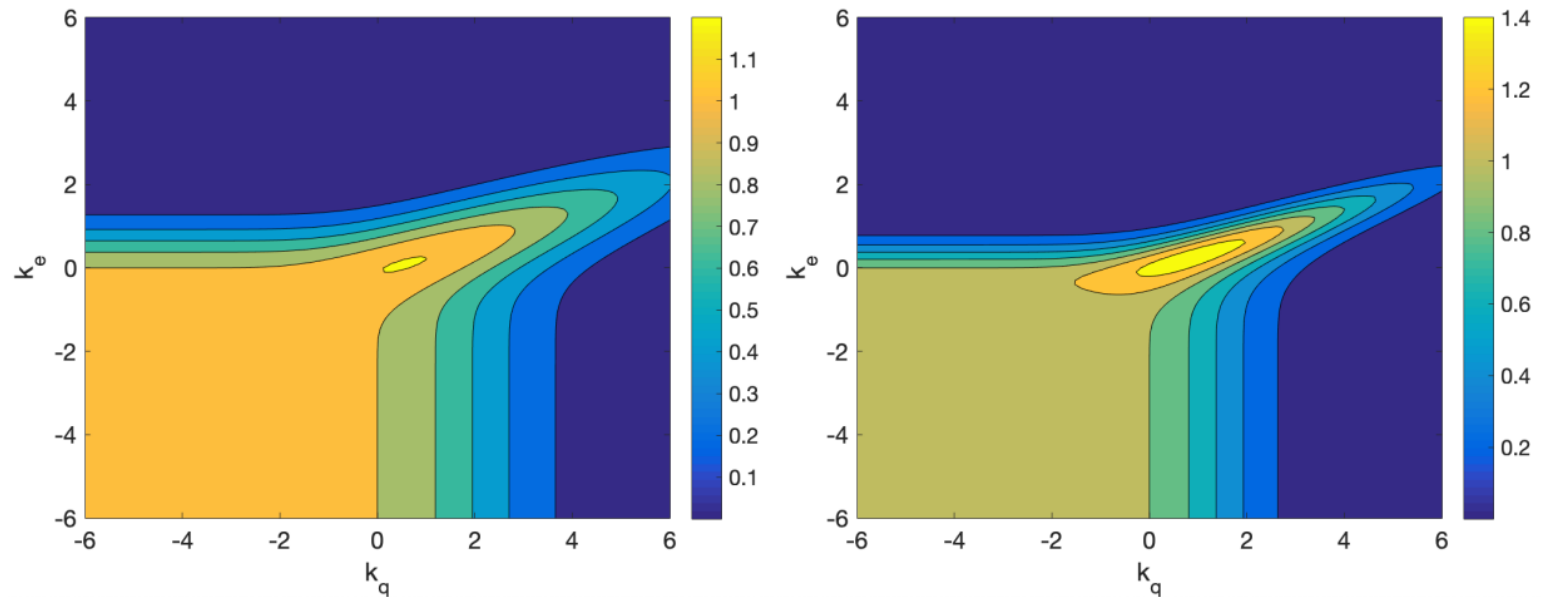

Figure 9. Ratio for $q_{1}$ for $\sigma_{2}$ and $\sigma_{3}$ inflation of 1.5 (left) and 3.0 (right)

These results show some increase in risk in the central region. However, firstly this inflation is minor and secondly as seen below, is not in the critical region where integrity bounding is achieved.

\section{CROSS-CORRELATION OBSERVATIONS}

Now that the impact of cross-correlation between position and test domains due to the measurement error model different from true error characteristic has been discussed through some canonical examples in previous sections, this section addresses some correlation observations through real GPS and Galileo measurement collection. We used dual-frequency GPS and Galileo observables from an ARAIM prototype designed by the Illinois Institute of Technology [18]. A total of 10 dates from August 08, 2019, to August 18, 2019, were investigated by assuming ARAIM implementation [15]. The position error and the solution separation test for ARAIM are respectively computed, and they are then normalized by the corresponding standard deviation of position error and that of the solution separation. The normalized position error, here represented as $P E$, can be computed as Equation (45).

$$
P E=\frac{x_{0}-x_{r e f}}{S_{0} \Sigma_{\varepsilon} S_{0}^{T}}
$$


Here the covariance, $\Sigma_{\varepsilon}$, is determined based on measurement noise variance for the continuity purpose in Equation (6). Also, the normalized solution separation test statistic is computed based on Equation (11).
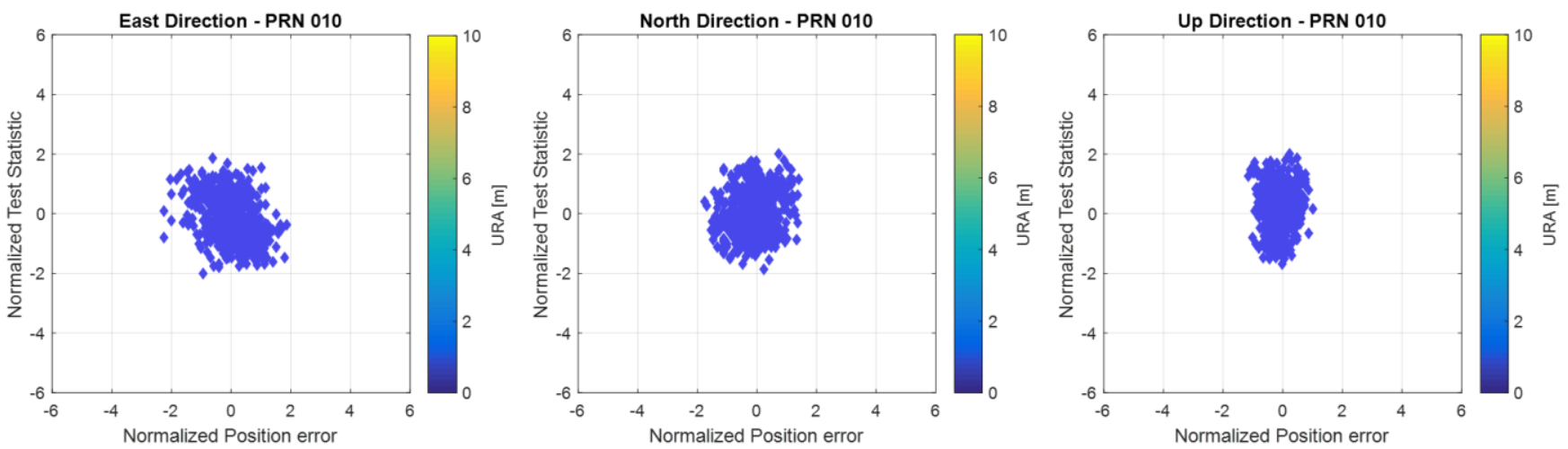

Figure 10. Distribution of normalized position error and solution separation test statistic under a single GPS satellite (PRN 10) fault. Position errors and test statistics are respectively normalized by corresponding standard deviations based on broadcast URA values.
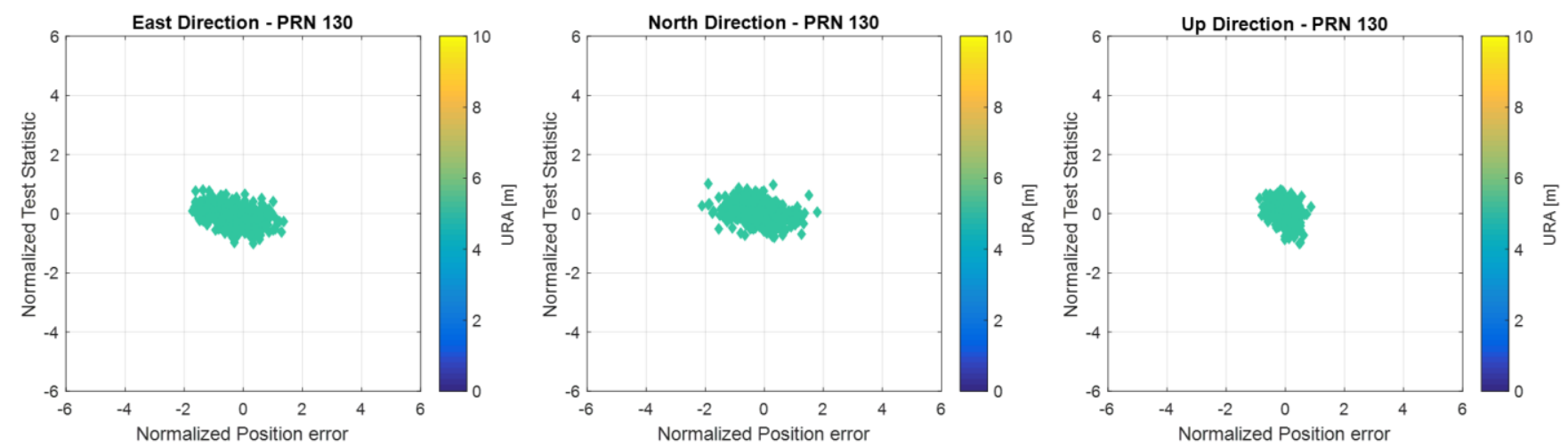

Figure 11. Normalized position error vs. normalized solution separation test statistic under a single Galileo satellite (PRN 130) fault
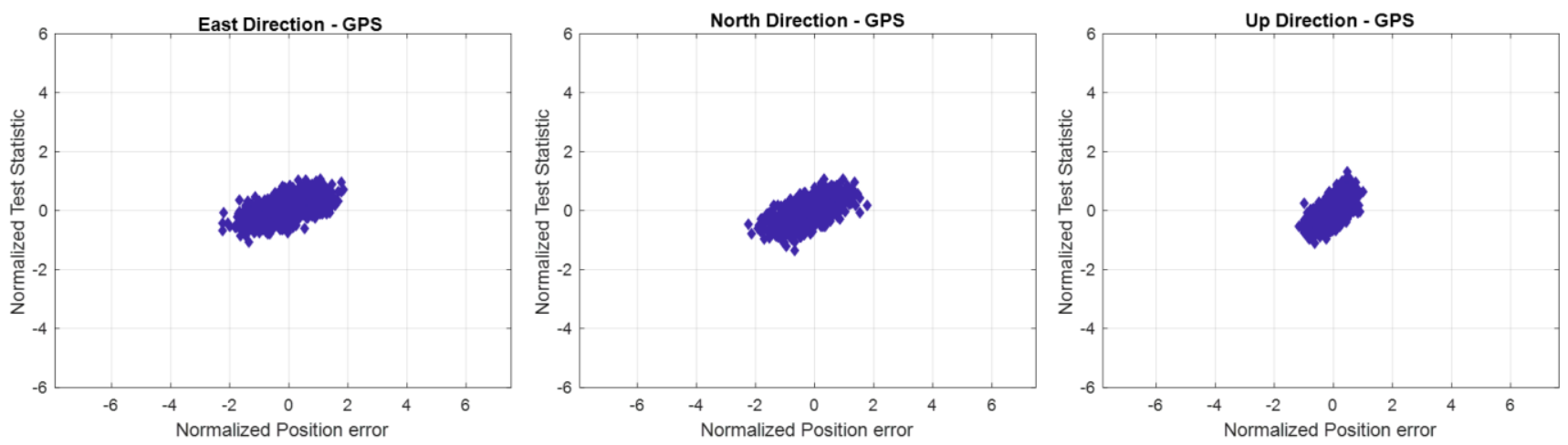

Figure 12. Population of normalized position error and solution separation test statistic under GPS constellation fault mode 

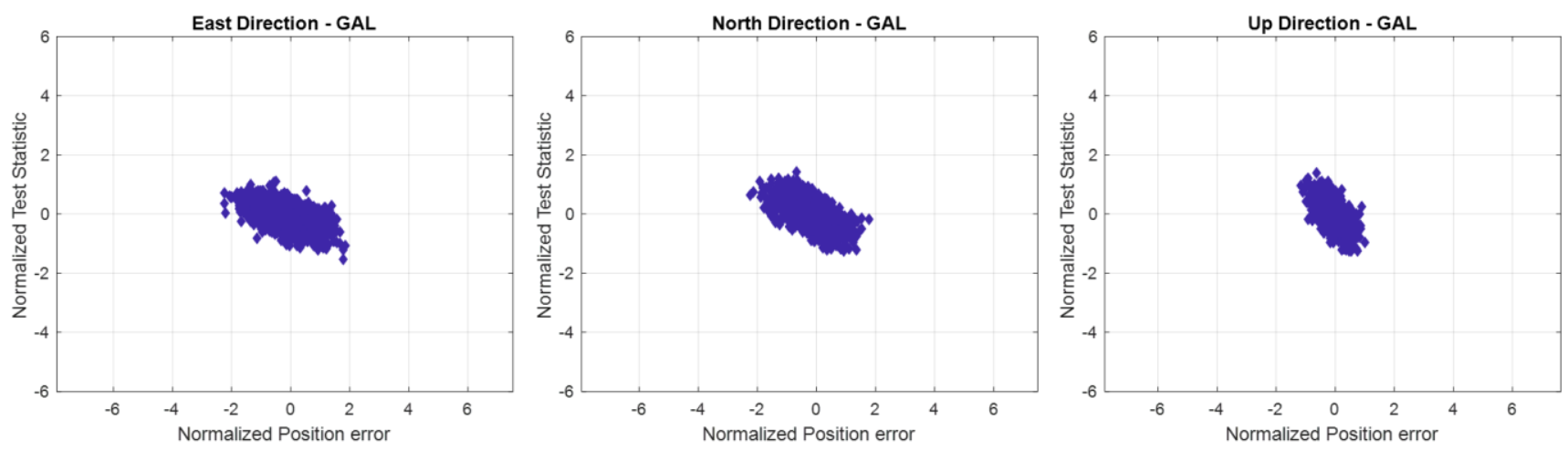

Figure 13. Normalized position error vs. normalized solution separation test statistic under Galileo constellation fault mode

Figure 10 and Figure 11 respectively show the population of the normalized position error and normalized solution separation test for a single GPS satellite fault and a single Galileo satellite fault for East, North and Up coordinates. Both GPS and Galileo measurements are used for the position estimation and test statistic computation, and broadcast URA values are used in the normalization. Each color bar indicates the URAs, and those URAs are approximately two meters for GPS satellites and about six meters for Galileo satellites in all coordinates. Similarly, distributions of the two normalized quantities for GPS and Galileo constellation fault modes are respectively shown in Figure 12 and Figure 13. Also, Table 1 summarizes the Pearson correlation coefficient [19] obtained from the scatter plot for each coordinate for the four different fault cases.

Table 1. Correlation coefficients for different four fault hypotheses

\begin{tabular}{c|c|c|c|c}
\hline Case & Fault mode & East & North & Up \\
\hline 1 & Single GPS fault & -0.39 & -0.12 & -0.07 \\
\hline 2 & Single Galileo fault & -0.37 & -0.34 & -0.30 \\
\hline 3 & GPS constellation fault & +0.59 & +0.63 & +0.60 \\
\hline 4 & Galileo constellation fault & -0.58 & -0.61 & -0.59 \\
\hline
\end{tabular}

In the table, non-zero correlation coefficients are observed for all fault modes examined. As described, since the modeled error standard deviations are different from true ones, the correlation between the position error and test statistic can be caused in the weighted least squares estimation process. In Figure 10 and the table, a higher correlation is present in the east direction compared to the other coordinates in the case of the GPS satellite fault. For the Galileo single satellite fault case (Figure 11 and Case 2 in Table 1), higher correlation coefficients are observed in all different directions than the GPS satellite fault case. In this case, the normalized distributions are tighter due to the higher URA (or SISA) values for Galileo which reduces the sensitivity of the position solution to Galileo measurements. This could explain the higher correlation after normalization if the Galileo URAs are overbounding with a greater margin. In addition, the strongest correlation effects are observed in the constellation fault mode tests (Figure 12 and 13, and Case 3 and 4 in Table 1). The different signs of the correlation between GPS and Galileo constellation faults are likely due to the higher Galileo SISA than the GPS URA. This has the result of the position solution being influenced more by the GPS measurements.

\section{REALISTIC GEOMETRIES AND GAUSSIAN INFLATION MODELS}

In this section, we further investigate the impact of the correlation due to the error model inflation on the integrity evaluation by taking into account 24 GPS/Galileo baseline constellation geometries for H-ARAIM applications [16] under which different combinations of sigma inflation are assumed. The correlation and risk ratios, defined in Equation (33), have been determined over different thresholds, $k_{e}$ and $k_{q}$ (See Section Canonical examples), for each satellite geometry by generating more realistic error 
inflation factors than previous canonical examples. For this purpose, GPS URA of $2.4 \mathrm{~m}$ is used for the overbounding error model, and URE values for different GPS satellites [17] and Galileo satellites [20] are considered for actual error model. More specifically, covariance matrices used in Equation (33) for the evaluation of the risk ratio are determined as follows. The original baseline ARAIM algorithm [15] determines the standard deviation of the position error using the error model for integrity purpose (See Equation (5)) and that of the solution separation test statistic with the error model for the accuracy and continuity purpose (See Equation (6)). However, for the simulation purpose, we use the URA value to compute the standard deviation for test statistic $\left(\sigma_{S S, U R A}\right)$ for the inflated case, and the corresponding covariance matrix $\left(\Sigma_{\varepsilon}\right)$ is shown in Equation (46).

$$
\boldsymbol{\Sigma}_{\varepsilon}=\left(\begin{array}{cc}
\sigma_{S S, U R A}^{2} & 0 \\
0 & \sigma_{o}^{2}
\end{array}\right)
$$

Similarly, we compute the standard deviation for position error $\left(\sigma_{O, U R E}\right)$ for the uninflated case by applying actual URE values obtained from previous investigations to different types of satellites, and the corresponding covariance matrix $\left(\Sigma_{\varepsilon}^{\prime}\right)$ is shown in Equation (47). Here the covariance term in the equation is derived using both URA and URE values, and $\rho_{\text {corr }}$ denotes the correlation coefficient.

$$
\boldsymbol{\Sigma}_{\varepsilon}^{\prime}=\left(\begin{array}{cc}
\sigma_{S S}^{2} & \rho_{c o r r} \sigma_{S S} \sigma_{0, U R E} \\
\rho_{c o r r} \sigma_{0, U R E} \sigma_{S S} & \sigma_{o, U R E}^{2}
\end{array}\right)
$$

Next, as performed in previous canonical examples (See Section Canonical examples), the multivariate normal cumulative density function with zero mean and covariances given by Equation (34) and (35) is evaluated for a set of $k_{e}$ and $k_{q}$ thresholds, ranging from -6 to 6 in steps of 0.1 units, in order to compute the risk ratio. An example result of the risk ratio for a satellite geometry is shown in Figure 14.

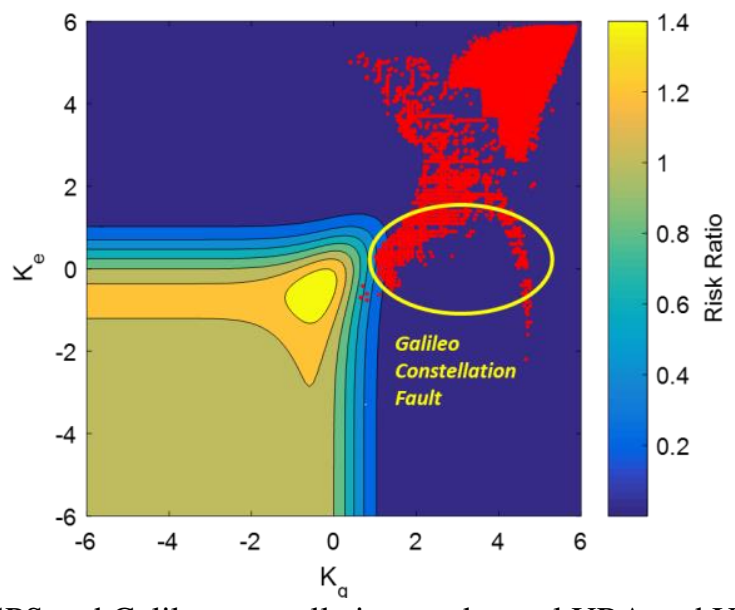

Figure 14. Risk ratio obtained when GPS and Galileo constellations and actual URA and UREs are applied and the population of normalized thresholds $k_{e}$ and $k_{q}$ simulated for H-ARAIM (red dots)

In this figure, the red dots show the distribution of the normalized thresholds, i.e., $k_{e}$ and $k_{q}$, that are computed based on 24 GPS and 24 Galileo constellations and error models for H-ARAIM applications. H-ARAIM availability simulation for 24 hours was carried out with formal parameters outlined in [16] to obtain the set of thresholds. As shown in the canonical examples, the risk ratio is less than one for all of the satellite geometries and fault modes generated through the H-ARAIM simulation. For the simulation, we used the Stanford Matlab Algorithm Availability Simulation Tool (MAAST) for ARAIM [21] with some modifications. Table 2 shows some key simulation parameters used in this section. In particular, the yellow circle indicates the Galileo constellation fault case, which is the dominant mode because its prior probability is currently set as $10^{-4}$ and corresponding all epoch risk estimation reaches up to approximately $10^{-3}$ from Figure 1 . 
Table 2 Key parameters for H-ARAIM simulation

\begin{tabular}{c|c|c|c}
\hline Parameter & Description & Parameter & Description \\
\hline Constellation & 24 GPS + 24 GAL & $b_{\text {nom }}$ & $0.75 \mathrm{~m}$ \\
\hline$\sigma_{U R E}$ & GPS [17] / GAL [20] & $\sigma_{U R A}$ & $2.4 \mathrm{~m}$ for GPS / GAL \\
\hline$P_{\text {sat }}$ & $10^{-5}$ & $P_{\text {const }}$ & GPS: $10^{-8} / \mathrm{GAL}: 10^{-4}$ \\
\hline Mask Angle & $5 \mathrm{deg}$. & User grid formation & 10 deg. by 10 deg. \\
\hline Simulation Time Step & $10 \mathrm{mins}$ & Simulation Duration & 24 hours \\
\hline
\end{tabular}

Therefore, we also examine how much impact the cross-correlation due to the conservative approximation of error model on the actual integrity risk evaluation by comparing the risk ratio distribution based on real GPS and Galileo measurements from the HARAIM prototype (See Section Cross-correlation observations) with the population of the simulated normalized thresholds, i.e., the red dots. The comparison is carried out for constellation fault cases. Figure 15 shows the results of the risk ratio obtained based on the experimental variance-covariance structure, i.e., variances and correlation coefficients, for the GPS and Galileo constellation fault conditions in Section Cross-correlation observations. The left one corresponds to the ratio for the Galileo constellation fault condition and the right one for the GPS constellation fault case. The figures show that even though the risk increases by up to $60 \%$ due to the correlation effect, such increase is only limited to some cases which are not critical for actual ARAIM geometries. Thus, the evidence suggests that for the assessment of the temporal correlation effect on integrity risk, the assumption of the independence between the position error and the test statistic would be acceptable.
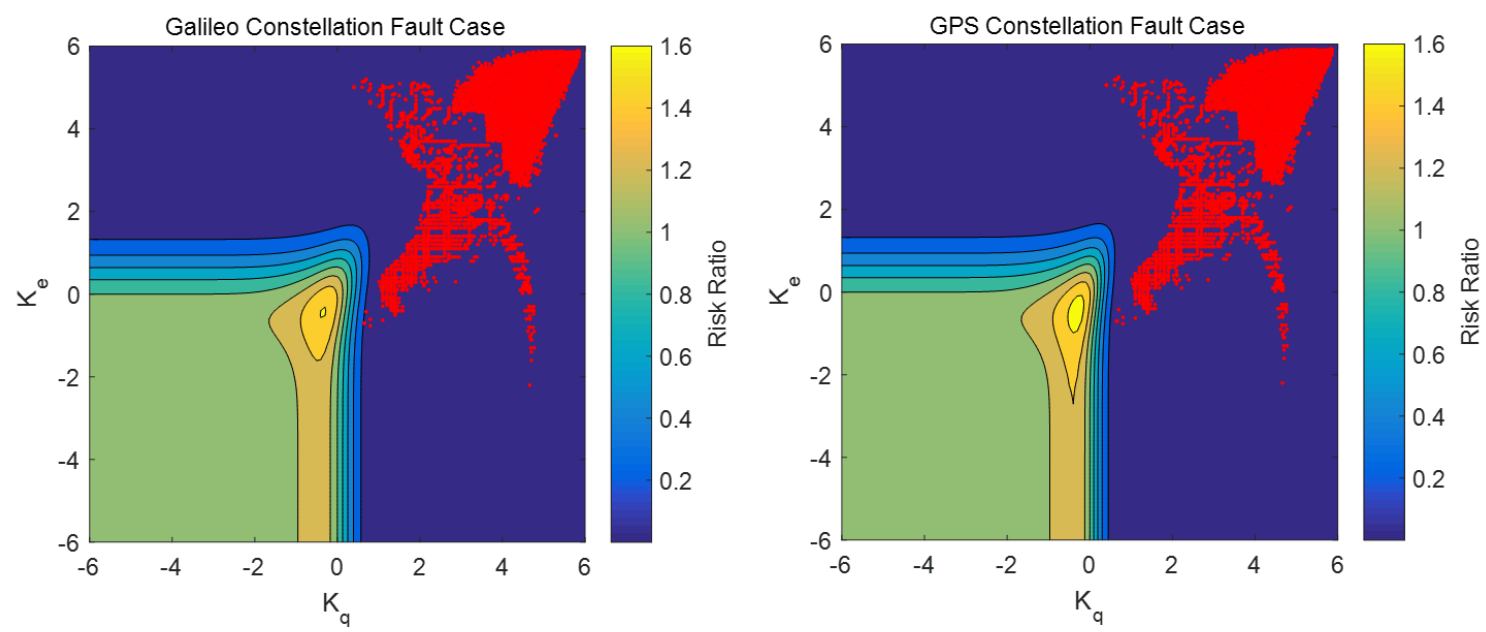

Figure 15. Risk ratio obtained based on real GPS and Galileo observables under constellation fault conditions and the distribution of normalized thresholds for H-ARAIM (red dots)

\section{CONCLUSION}

In this paper, we have taken a number of approaches to evaluate the level of correlation between the position errors and the fault detection statistics for ARAIM and also to determine its impact on the integrity risk. Additional integrity risk was observed: maximum $10 \%$ increase for some canonical examples, up to $40 \%$ increase for simulated H-ARAIM geometries and error models, and the maximum $60 \%$ increase for H-ARAIM prototype observations. However, it appears that the impact of cross-correlation observed is only present for high probabilities of missed detection which are not those employed by the previously proposed algorithm assessing 
the number of effective samples in paired process case [1]. We conclude therefore that the assumption taken in that assessment is valid with respect to correlation resulting from sigma inflation.

Our findings on the correlation effect might be extended to wider scientific applications such as recent developments in innovation monitoring in Kalman Filter [22] where the independence of the position and test statistic domains is often assumed. Correlation can also be introduced by using alternative optimized methods for the position solution [23]. Thus, work on the correlation impact in that case has been ongoing, where initial tests showed a stronger correlation effect. Further work in this regard is needed.

\section{ACKNOWLEDGMENTS}

This work is conducted within the framework of a cooperation agreement between ENAC and DSNA/DTI for ENAC to provide scientific support to DTI on GNSS. The authors would like to thank the DSNA for their financial support of this work.

\section{REFERENCES}

References should be numbered consecutively in the text with numbers in brackets, and appear at the end of the paper in the format shown below:

1. E. Bang, C. Milner, C. Macabiau and P. Estival, "Sample Temporal Correlation Effect on PHMI," Proceedings of the 2019 International Technical Meeting of The Institute of Navigation, Reston, Virginia, January 2019, pp. 85-99.

2. RTCA, “DO 316 - Minimum Operational Performance Standards (MOPS) for GPS/ABAS Airborne Equipment," DO 316, Apr. 2009.

3. B. Parkinson and P. Axelrad, "A Practical Algorithm for Autonomous Integrity Verification Using the Pseudo Range Residual," NAVIGATION, Journal of the Institute of Navigation, 1988.

4. M. Sturza, "Navigation System Integrity Monitoring Using Redundant Measurements," Navig. J. Inst. Navig., vol. 35, no. 4, pp. 483-502, 1989.

5. G. R. Brown and P. W. McBurney, “Self-contained GPS Integrity Check Using Maximum Solution Separation,” Navig. J. Inst. Navig., 1988.

6. R. Kelly, "The Linear Model, RNP, and the Near-Optimum Fault Detection and Exclusion Algorithm," Navig. J. Inst. Navig., 1998.

7. C. Milner, "Navigation Integrity for Civil Aviation," INPT, 2017.

8. T. Walter and P. Enge, "Weighted RAIM," in Proceedings of ION GPS, 1995.

9. G. R. Brown and Y. G. Chin, "GPS RAIM: Calculation of thresholds and protection radius using Chi-Square methods - A geometric approach,” Navig. J. Inst. Navig., 1998.

10. R. Young and G. McGraw, "Fault Detection and Exclusion Using Normalized Solution Separation and Residual Monitoring Methods," in 15th International Technical Meeting of The Satellite Division of the Institute of Navigation, Portland, Oregon, 2002, vol. Volume 50.

11. B. S. Pervan, S. P. Pullen, and J. R. Christie, “A multiple hypothesis approach to satellite navigation integrity,” Navigation, vol. 45, no. 1, pp. 61-71, 1998.

12. Y. C. Lee and M. P. McLaughlin, "Feasibility analysis of RAIM to provide LPV-200 approaches with future GPS," in ION GNSS 20th International Technical Meeting of the Satellite Division, 2007, pp. 2898-2919.

13. J. Blanch, A. Ene, T. Walter, and P. Enge, "An Optimized Multiple Hypothesis RAIM Algorithm forVertical Guidance," in 20th International Technical Meeting of The Satellite Division of the Institute of Navigation, Fort Worth, Texas, 2007.

14. M. Joerger, F.-C. Chan, and B. Pervan, "Solution Separation Versus Residual-Based RAIM: Solution Separation Versus Residual-Based RAIM," Navigation, vol. 61, no. 4, pp. 273-291, Dec. 2014.

15. J. Blanch et al., "Baseline Advanced RAIM User Algorithm and Possible Improvements," IEEE Trans Aerosp. Electron. Syst., vol. 51, no. 1, pp. 713-732, Jan. 2015. 
16. WG-C, "EU-US Cooperation on Satellite Navigation Working Group C ARAIM Technical Subgroup Milestone Report 3." Feb2016.

17. Walter, Todd, Blanch, Juan, "KEYNOTE - Characterization of GNSS Clock and Ephemeris Errors to Support ARAIM," Proceedings of the ION 2015 Pacific PNT Meeting, Honolulu, Hawaii, April 2015, pp. 920-931.

18. Cassel, R.,"Real-time ARAIM using GPS, GLONASS, and GALILEO", M.S. Thesis, Department of Mechanical, Materials and Aerospace Engineering, Illinois Institute of Technology, May 2017.

19. A. Papoulis and S.U. Pillai, Probability, Random Variables, and Stochastic Processes, McGraw-Hill, $4^{\text {th }}$ edition, 2002.

20. Perea, S. et al. "URA/SISA Analysis for GPS and Galileo to Support ARAIM," NAVIGATION, Vol. 64, No. 2, 2017, pp. 237 254.

21. ARAIM Matlab Algorithm Availability Simulation Tool (MAAST), https://gps.stanford.edu/resources/tools/maast.

22. Tanil, C., Khanafseh S. Joerger M., and Pervan B., "An INS Monitor to Detect GNSS Spoofers Capable of Tracking Vehicle Position,” IEEE Trans Aerosp. Electron. Syst., Vol. 54, No. 1, pp. 131-143, Feb. 2018.

23. J. Blanch, T. Walter, P. Enge and V. Kropp, "A Simple Position Estimator That Improves Advanced RAIM Performance," IEEE Trans Aerosp. Electron. Syst., vol. 51, no. 3, pp. 2485 - 2489, July 2015. 